\title{
La scalarité aux fondements de la distinction entre constructions équative et similative?
}

\author{
Pascale Hadermann, Michel Pierrard, Dan Van Raemdonck \& Valerie Wielemans ${ }^{1}$ \\ Universiteit Gent, Vrije Universiteit Brussel, Université Libre de Bruxelles, FWO Vlaanderen \\ pascale.hadermann@ugent.be, mpierrar@vub.ac.be, dvanraem@ulb.ac.be, valerie.wielemans@ugent.be
}

Le terme « scalaire » réfère communément à une échelle de grandeurs, de degrés, c'est-à-dire à une série, une suite continue ou progressive de niveaux constituant une hiérarchie dans un domaine donné. De plus, comme la notion d'échelle est souvent appréhendée de façon sérielle, elle sera en conséquence aussi considérée comme étant orientée. Cette caractérisation du concept de scalarité le rend particulièrement apte à traiter de phénomènes relevant de domaines variés, allant de la quantification et de la gradation, à la comparaison ou à l'intensification. Une représentation scalaire sous-tendrait alors des manifestations discursives spécifiques dans l'ensemble de ces contextes et induirait en conséquence un traitement unitaire d'une large gamme de structures à première vue fort divergentes, en français mais aussi dans une grande variété d'autres langues.

L'objectif de la présente contribution est, plus modestement, d'évaluer, à partir des données du français, en quelle mesure la scalarité est pertinente pour opérer une distinction entre les deux types de structures qui, dans de nombreuses langues d'Europe en particulier ${ }^{2}$, sont associés à la comparaison d'égalité : les constructions équatives et similatives (cf. Haspelmath \& Buchholz 1998). Ces constructions sont respectivement illustrées par les exemples français (1a) et (2a), auxquels sont jointes les paraphrases de Haspelmath \& Buchholz (1998: 278). Celles-ci visent à expliciter au niveau sémantique la base commune et les différences entre les deux tours :

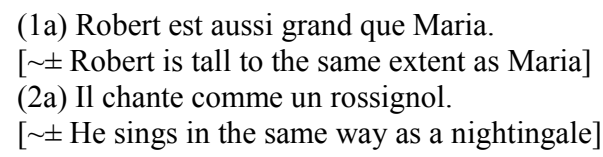

\section{Egalité scalaire et non scalaire.}

1.1. Certaines observations dans des études récentes concernant la différence entre ces deux types de constructions et leurs marqueurs respectifs pourraient laisser penser que c'est précisément la propriété scalaire qui permet de discriminer entre tours équatifs et tours similatifs :

- « equatives express equal extent, similatives express equal manner » (Haspelmath \& Buchholz 1998 : 278);

- «Scalar comparison involves grading, while non-scalar comparison is concerned with identity and likeness » (Huddleston et al. 2002 in Buzarovska 2005).

- «Equivalence acts as a framework domain that subsumes both equality and similarity. The difference between these two concepts depends on the presence of a quantifying or non-quantifying identity operator in the semantic representation of comparative as- and like-clauses » (Buzarovska $2005: 75$ )

Pour ces auteurs, les équatives exprimeraient en quelque sorte une égalité scalaire, liée aux notions de quantité, de quantification ou de gradation, tandis que les similatives proposeraient une égalité non scalaire, rattachée au non quantitatif, à la manière, à la similarité.

Cependant, un examen plus détaillé du fonctionnement des deux types de marqueurs impliqués en (1a) et (2a) ((aus)si...(que) et comme) révèle qu'ils peuvent tous les deux en français indiquer le haut degré :

(1b) Il est si grand !

(2b) Comme il chante bien ${ }^{3}$ ! 
Faut-il dès lors conclure que les notions de scalarité et de haut degré ne se recouvrent pas puisque les marqueurs équatifs 'scalaires' et les marqueurs similatifs 'non scalaires' peuvent marquer ce dernier ? $\mathrm{Ou}$, au contraire, la scalarité ne séparerait-elle pas aussi radicalement qu'on le prétend les équatives et les similatives puisque leurs marqueurs respectifs marquent le haut degré dans un autre type de constructions, les exclamatives ${ }^{4}$ ?

1.2. L'examen de l'impact de la scalarité sur les deux constructions demande d'abord une définition plus resserrée du concept. En partant d'une caractérisation commune de celui-ci (cf. Hadermann, Pierrard \& Van Raemdonck 2007), nous appréhendons la scalarité comme une opération d'envisagement, au moyen de marqueurs linguistiques, de propriétés ou d'états sur une échelle quantitative ou qualitative :

a) l'opération d'envisagement peut viser au positionnement de ces propriétés ou états sur l'échelle, ce qui impliquera aussi un certain ordre (cf. aussi Tovena 2007). Ce positionnement peut être relatif (envisagement de l'écart entre deux propriétés/ états ${ }^{5}:$ il est plus fort que moi; il est fort comme un turc) ou absolu (un(e) seul(e) des deux états/ propriétés est actualisé(e) : il est le plus fort). De manière intermédiaire, l'opération peut enfin concerner l'envisagement du rapport de proportionnalité entre deux propriétés/ états (plus il s'entraîne, plus il gagne);

b) elle peut aussi dans certains cas simplement se limiter à marquer une orientation (cf. Bat Zeev 2007) vers le haut ou le bas degré (comme elle mange beaucoup/ peu!; elle mange énormément/ presque rien).

En conséquence, une approche plus fine de la notion demande à distinguer deux niveaux de scalarité, un niveau plus indéterminé qui se limite à une orientation vers le haut ou le bas degré (scalB) et un niveau plus spécifié qui précise un positionnement sur une échelle (scalA).

1.3. Il découle de la distinction que nous venons d'introduire ci-dessus qu'on ne peut confondre le marquage de la scalarité et celui de la quantification ${ }^{6}$. Ainsi, si certains marqueurs scalaires ((au)tant, plus et moins) peuvent occuper une position de quantificateur (3a), ce n'est certainement pas le cas de tous (3b) :

(3a) Beaucoup/ autant/ plus/ moins de gens n'étaient pas prévus à ce concert.

(3b) *(aus)si de gens n'étaient pas prévus à ce concert.

D'autre part, certains marqueurs scalaires peuvent se combiner avec un quantifiant, contrairement à d'autres :

(4a) Il a mangé aussi peu que Pierre/ *Il a mangé autant peu que Pierre

(4b) Il a si peu dormi/ *Il a tant peu dormi !

Certains marqueurs scalaires quantifiants sont en réalité des formes synthétiques combinant un marqueur scalaire et un quantifiant :

(5a) Il a mangé autant [= aussi beaucoup] que Pierre

(5b) Il a tant [= si beaucoup] dormi !

D'un point de vue typologique, c'est une caractéristique de nombreuses langues européennes d'avoir « special words incorporating both the notion of quantity and the marking of the parameter » (Hapelmath \& Buchholz $1998: 298)$. Si les notions de quantification et de scalarité se touchent et se recouvrent même partiellement (5a-b), il est cependant nécessaire de caractériser plus précisément leur fonctionnement. Le trait [+degré] n'est pas identique à [+quantifiant] et, en ce qui concerne les deux types de scalarité répertoriés ci-dessus en particulier, il importe de ne pas confondre les marqueurs [+quant] et [-quant] : ainsi, pour scalA, le trait [-/+quant] permettra de spécifier la différence aussi/ autant (1a-5a), et pour scalB, celle entre si/ tant $(1 \mathrm{~b}-5 \mathrm{~b})$ :

(1a) Robert est aussi grand que Maria.

(1b) Il est si grand !

(5a) Il a mangé autant que Pierre

(5b) Il a tant dormi ! 
Après avoir mieux délimité et affiné la notion de scalarité, l'étude tentera de préciser son rôle dans le'appréhension du fonctionnement des diverses constructions - équatives, similatives et exclamatives introduites par les marqueurs (aus)si et comme. Pour ce faire, elle procédera en trois étapes : (a) d'abord, décrire les lectures scalaires des diverses constructions afin de spécifier le type de scalarité convoqué ; (b) ensuite, déterminer la manière dont ces lectures scalaires sont générées dans les diverses constructions ; (c) enfin, caractériser plus finement le fonctionnement des marqueurs si, aussi que et comme.

\section{La scalarité dans les constructions en (aus)si...(que) et en comme}

La description du fonctionnement des constructions en [aus]si [que] et en comme doit permettre d'identifier les diverses interprétations scalaires dans chacun de ces tours.

\subsection{Interprétations scalaires dans les constructions en si}

Nous observerons successivement l'interprétation des constructions (1a) comprenant aussi que (si+) et celles contenant uniquement si (1b).

\subsubsection{Si+}

Aussi...que indique habituellement la position relative des comparandes sur une échelle scalaire (scalA). Les comparandes dont on pose l'équivalence sur l'échelle scalaire, seront des arguments (6a), des «adverbiaux » $(6 \mathrm{~b})$ ou des prédicat(ion)s $(6 \mathrm{c}-\mathrm{d})$ :
(6a) Il dépense de l'argent aussi vite que sa femme.
(6b) Il a couru aujourd'hui aussi vite qu'hier.
(6c) Il dépense l'argent aussi vite qu'elle le gagne.
(6d) Il dépense l'argent aussi vite qu'elle le lui permet.

Dans des contextes spécifiques, il peut pourtant être orienté vers une lecture moins définie qui se limite à marquer l'orientation (scalB). Ainsi, lorsqu'il n'y a pas d'étalon d'exprimé, si+ en arrive, par défaut de pôle d'équivalence, à exprimer pragmatiquement une orientation vers le haut degré :

(7a) Elle est ressortie, toujours aussi pâle, bien que sa joue droite porte la marque rouge de cinq doigts.

(7b) Pourquoi cette prose est-elle de bout en bout, sans présenter de qualités formelles bien apparentes, à la fois aussi intensément vivante et aussi intimement "personnalisée" ?

Le maintien de l'interprétation comparative est lié, d'après Milner (1978 : 366-369), à la capacité, grâce aux informations disponibles (dans le contexte ou dans la connaissance du monde), à disjoindre référentiellement le comparé et l'étalon. Lorsque ceux-ci ne peuvent être disjoint, l'interprétation s'oriente vers le haut degré :

(8a) Jeanne est ressortie toujours aussi pâle (qu'elle était) ${ }^{7}$.

$=>$ interprétation intensive

(8b) Après Jeanne, Nicole est ressortie aussi pâle (que Jeanne était).

$=>$ interprétation comparative

\subsubsection{Si}

$S i$ n'envisage pas un positionnement sur l'échelle mais une orientation vers le haut degré. C'est un marqueur scalB.Par ailleurs, il ne marque pas la quantification mais l'intensité [-quant], ce qui explique la différence dans le fonctionnement avec très, qui est par contre [+quant] :

(9a) Notre prof est très/ si correct.

(9b) Il avance très/ si lentement.

(9c) Cette maison est ??très/ si immense ; son refus de dialoguer est ??très/ si total. 
(9d) Il est très/ * si exactement douze heures.

Très exprime l'orientation vers le haut de l'échelle, mais a une valeur quantifiante 'objective' (cf. aussi Milner 1978 ; Rivara 1990) et se combine donc mal avec des adjectifs/ adverbes qui indiquent déjà par leur sémantisme cette valeur $(9 \mathrm{c})$. De son côté, si exprime aussi une orientation vers le haut degré, mais a une valeur non quantifiante, intensive, qui lui permet de 'subjectiviser' ce type d'adjectfs/ d'adverbes, et donc de rendre l'énoncé acceptable. Par contre, si ne fonctionne pas dans l'expression du haut degré quantitatif 'objectif' (9d).

\subsection{Interprétations scalaires dans les constructions en comme}

Nous discuterons successivement les interprétations des tours $2 \mathrm{a}$ (comme1) et $2 \mathrm{~b}$ (comme2).

\subsubsection{Comme1}

Comme indique ici la manière et est donc normalement [- scal]

(10a) Il ment comme il respire.

(10b) Il a dépensé l'argent comme je le lui ai conseillé

Il ne produit alors aucune valeur de degré, mais est l'expression d'une 'manière partagée'. Dans le prolongement de cette valeur 'qualitative', il y a le comme de conformité, où la 'manière partagée' devient une vague conformité entre prédications (10c: "ainsi que"/ "de même (que)"), ou encore le comme additif, avec les deux comparandes partageant le même prédicat (10d) :

(10c) Comme les amateurs de vin parlent en millésimes, les Anglais mesurent la valeur de leur jeunesse universitaire aux performances des équipages.

(10d) Jean comme Paul a les yeux bleus.

Que commel ne participe pas en soi à l'expression du degré est illustré par sa capacité à comparer un comparé et un étalon qui s'opposent par leurs propriétés, ce que l'égalité ou l'inégalité sont incapables de faire (Muller $1996: 248)$ :

(11a) Il est intelligent comme son frère ne l'est pas.

(11b) *Il est aussi/ plus/ moins intelligent que son frère ne l'est pas.

Certains contextes, toutefois, peuvent amener à une lecture scalaire de comme1 :

a) Lorsque la conformité des prédicats comparés induit une interprétation quantitative, le marqueur devient alors l'expression de scalA et induit une égalité quantitative (=autant)

(12a) Il mange comme tu bois .
(= il mange autant que tu bois)

b) Dans d'autres configurations, commel peut également exprimer scalB. C'est le cas lorsque l'étalon apparaît comme le parangon par rapport au repère de la comparaison (12b,c : valeur prototypique/ stéréotypique), lorsque le repère est une qualité dont on présuppose que l'étalon la possède (ou ne la possède pas) à un haut degré par essence (12d : valeur contextuelle) :

(12b) Il est fort comme un turc.

(12c) Il chante comme un rossignol.

(12d) Il est courageux comme ton cousin.

C'est également une interprétation scalB qui est induite dans (11a) de l'absence de qualité partagée ou de conformité entre le comparé et l'étalon, de sorte que le degré de la qualité attribuée au comparé n'est plus limité. 


\subsubsection{Comme2}

Déjà, la transformation de l'étalon en parangon ouvrait la voie à scalB. Ce sera d'autant plus le cas lorsque tout contexte d'étalonnage du prédicat repère disparait ${ }^{8}$. Logiquement, comme2 induit dans de tels cas une orientation vers le haut degré :

(13a) Comme c'est beau! / Comme tu travailles! / Comme tu travailles bien!

(13b) Comme tu dis des bêtises aujourd'hui!

Il exprime donc un effet de sens scalB. Relevons qu'il n'a plus ici la valeur d'une proforme de manière, puisqu'il ne peut plus en français moderne fonctionner en interrogative, ni pour questionner sur la manière, ni d'ailleurs sur la quantité :

(13c) Comment $(*$ comme) sont-ils partis ?

(13d) Combien $(*$ comme) sont partis?

\section{L'engendrement des valeurs scalaires}

Puisque les divers tours en si et en comme, les similatifs, les équatifs et les exclamatifs, permettent des lectures scalaires, il reste à déterminer si elles sont générées de la même manière. Bref, comment les diverses constructions en arrivent-elles à exprimer la scalarité ?

\subsection{Différents types d'effets scalaires}

La description des différentes interprétations scalaires dans le point précédent nous apprend qu'il y a non seulement d'un point de vue de la valeur sémantique deux types de scalarité (scalA/scalB) mais que, du point de vue syntaxo-discursif également, il faut distinguer deux manières d'attribuer aux constructions examinées ici une lecture scalaire.

a) La lecture scalaire est inhérente à la construction : elle sera le produit direct d'une des composantes de celle-ci, plus spécifiquement d'un marqueur spécifique :

- l'interprétation scalA de si+ dans les énoncés (6) est liée aux propriétés du marqueur équatif de la construction :

(14a) Paul est grand et tu l'es aussi > tu es aussi grand que Paul.

- l'interprétation scalB de si dans les énoncés (9) est également liée aux propriétés du marqueur de degré de la construction:

(14b) Tu travailles vite $>$ Tu travailles $s i$ vite !

b) La lecture scalaire peut aussi être non inhérente à la construction et être le produit d'une interaction avec le contexte syntaxo-discursif. Elle sera déclenchée par exemple par la présence ou au contraire l'effacement d'un élément spécifique de l'énoncé. Voyons comment la présence ou au contraire la suppression de certains éléments de l'énoncé peuvent engendrer une telle lecture scalaire :

- l'effet scalB de $s i+$ dans (7a-b) est produit par l'impossibilité d'identifier, sur la base de l'information disponible, un étalon conceptuellement disjoint du comparé ;

- les lectures scalA et scalB de commel dans (12a-d), sont liées quant à elles aux types de prédicats des comparandes ou aux propriétés des étalons ;

- enfin, l'interprétation scalB de comme2 est l'expression de la disparition de tout contexte d'étalonnage du prédicat repère (13a-b).

Examinons à présent plus précisément comment ces différentes interprétations scalaires et leur génération permettent d'appréhender le fonctionnement des différentes constructions comparatives ou exclamatives ainsi que celui de leurs marqueurs respectifs? 


\subsection{Scalarité et comparaison}

Plusieurs types de constructions se centrent sur le rapport entre un comparé et un étalon (15a-b;16a-b) et permettent par ailleurs une interprétation scalaire (6a-d ; 12a-c) :

$$
\begin{aligned}
& \text { (15a) Paul est aussi fort que son frère. } \\
& \text { (15b) Paul court aussi vite que son frère. } \\
& \text { (16a) Paul est fort comme son frère. } \\
& \text { (16b) Paul court vite comme son frère. }
\end{aligned}
$$

Leur manière de marquer la comparaison et d'inférer des lectures scalaires les différencient toutefois nettement.

\subsubsection{Comparatives en aussi...que}

L'énoncé (15a) signifie que "Paul est fort à un certain degré, égal à celui de son frère". Si y marque une orientation vers le haut degré et au(tre) ... que borne l'orientation en positionnant la qualité sur l'échelle au même degré de 'l'autre'.

a) La scalarité trouve bien sa source dans l'opérateur si+ : celui-ci n'est pas combinable avec d'autres indices du haut degré (15c) ou de positionnement au sommet de l'échelle (15d)

$(15 \mathrm{c}) *$ Paul est aussi \{très, trop $\}$ fort que son frère.

(15d) *Lors du concours d'éloquence, Paul était aussi le plus brillant que son frère il y a quelques années.

b) L'opérateur scalaire $s i$ 'complété' établit en outre une gradation relative de deux éléments sur l'échelle de propriété adjectivale/ adverbiale. Cette opération, qui implique en réalité deux marqueurs (aussi et $q u e$ ), est le fruit d'un processus de grammaticalisation complexe. Ce qui dans une langue comme le français a été grammaticalisé à travers l'histoire de la structuration et de la restructuration des systèmes, est explicité d'une manière remarquable dans des créoles à base française des Seychelles (Haspelmath \& Buchholz 1997 : 284) :

(17) I ris meme degre ki nu.

[Il est aussi riche que nous]

Effectivement, en résumant l'évolution du marqueur équatifs en français (et en faisant abstraction d'une série de phénomènes de réanalyse de structures ou de marqueurs et de réorganisation de systèmes), on peut relever les types de fonctionnement suivants (cf. Pierrard 1998 et 2002 et déjà Muller 1996) :

- jusqu'à la fin du moyen français, la comparaison d'égalité était marquée par la structure si/ tant $X$ comme (exemples tirés de Jonas 1971) :

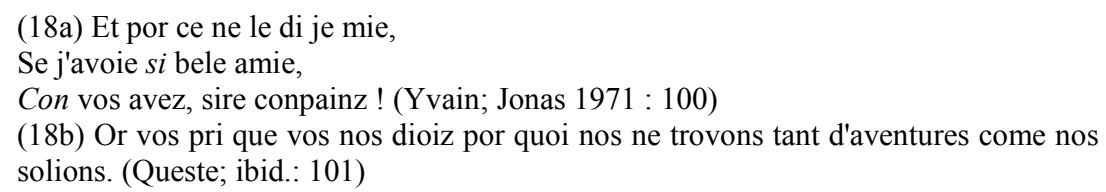

Si nous séparons l'apport du marqueur du repère (MRep) et celui du marqueur de l'étalon (MEtal) dans l'élaboration de la construction de l'ancien français, leur rôle sera décrit de la manière suivante :

MRep sil tant : marquage d'orientation de degré ;

MEtal com : affirmation de similarité de degré pour l'étalon (cf. le néerlandais zo ...als).

- dès le moyen français, une autre structure se développe : au(tre)si/autant X que, où le rôle des deux marqueurs change :

(19a) J'ai une aussi belle amie que vous.

(19b) Vous ferez autant d'expériences que vous voudrez. 
MRep aussi : l'orientation de degré ET l'annonce du même degré chez un autre comparande sont concentrées dans le premier marqueur ;

MEtal que : le deuxième élément se limite à introduire l'étalon avec lequel on pose l'égalité sur une échelle, ce qui permet l'alignement sur les systèmes de la comparaison de supériorité/ d'infériorité (plus/ moins/ aussi beau que).

c) La scalarité inhérente à la construction est par ailleurs, communicativement parlant, au centre de l'énoncé : pragmatiquement parlant en effet, «the speaker asserts a certain degree of likeness between $\mathrm{x}$ and $\mathrm{y}$ relative to some shared property. The existence of a common property in $\mathrm{x}$ and $\mathrm{y}$ is presupposed in the equality comparison, while the measured degree of likeness is new » (Buzarovska 2005 : 77).

D'une certaine manière, on peut affirmer que la nouvelle structuration de l'opérateur scalaire souligne ceci, en concentrant l'information concernant « the measured degree of likeness » sur MRep -rendant l'équativité du repère saillant - et réduisant MEtal à une marque de dépendance (que) servant à délimiter l'étalon auquel la mesure s'applique.

\subsubsection{Comparatives en comme}

L'énoncé (16a) signifie que "Paul est fort d'une manière comparable à ce que son frère est". Comme y marque la similarité d'une qualité donnée.

a) Le marqueur comme n'indique pas d'orientation ou de degré sur une échelle (16c). Ceci est confirmé par le fait qu'il est combinable avec des indices du haut ou du bas degré (16d) et de positionnement sur l'échelle (16e) :

(16c) Il mange comme Paul, un yaourt par jour (= peu)/ trois steaks par repas (= beaucoup).

(16d) Paul est très/ trop fort comme son frère.

(16e) Lors du concours d'éloquence, Paul était le plus brillant, comme son frère il y a

quelques années.

Lorsque l'énoncé en comme permet malgré tout une interprétation scalaire, c'est le type de prédicat comparé (16f) dans le cas de l'interprétation scalA ou l'étalon/ parangon (16g) dans le cas de scalB, qui oriente vers une lecture scalaire et définit l'orientation de celle-ci :

(16f) Il a bu comme tu as bu. [+quant]

(16g) Il est fort comme un Turc. (haut degré)/ généreux comme un Ecossais. (bas degré)

b) Le caractère non inhérent de la valeur scalaire est souligné par le fait que, d'un point de vue communicatif, la scalarité est secondaire puisque c'est la similarité du comparé et de l'étalon qui est au centre de l'énoncé : pragmatiquement parlant en effet, «the speaker asserts similarity of the referent $\mathrm{x}$ with y by way of comparing some common property whose existence is presupposed in y but is new information in $\mathrm{x}$. Hence, similarity comparison functions as a grounding strategy for $\mathrm{x}$ by assessing likeness between $\mathrm{x}$ and $\mathrm{y} \gg$ (Buzarovska 2005 : 77).

C'est donc de manière tout à fait conséquente que le marqueur similatif est purement un MEtal, focalisant l'attention sur l'étalon, tandis que le repère reste non marqué.

c) La saillance de l'étalon et le renvoi au second plan du repère est une caractéristique centrale qui explique la genèse de toute une série de tours en commel, une tendance générale dans les langues d'Europe (cf. Hapelmath \& Buchholz 1998 : 319-324).

- ainsi, le renvoi au second plan du repère conduit aux tours exprimant la conformité. Ici, le repère devient totalement implicite (10c ; les 'similes' chez Hapelmath \& Buchholz 1998 : 319) et ce qui est asserté est simplement la conformité du comparé et de l'étalon :

(10c) Comme les amateurs de vin parlent en millésimes, les Anglais mesurent la valeur de leur jeunesse universitaire aux performances des équipages. 
- une autre conséquence du centrage sur la similarité avec l'étalon et non pas sur la gradation du repère est la possibilité de centrer le focus communicatif sur la «new information in x » (cf (16a) : Paul est fort; (16b) : Paul court vite), dont le sens est déterminé au moyen de la similarité avec l'étalon, en position additionnelle (= de même y) et induisant une lecture non scalaire, un type de fonctionnement qui est impossible dans le cas des équatifs $\left(15 \mathrm{a}^{\prime}-\mathrm{b}^{\prime}\right)$ :

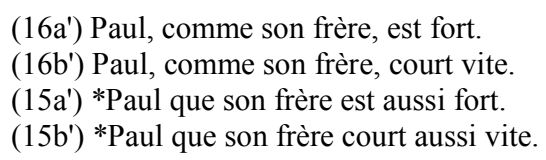

- La saillance de l'étalon au détriment du repère explique enfin le développement du marqueur similatif dans toute une série de tours non comparatifs, tels les phrases 'illocutives' (20a) et les 'énoncés exprimant un rôle' (20b)

(20a) Comme les scientifiques l'ont prédit, le réchauffement de l'atmosphère fait fondre les glaciers.

(20b) Il travaille comme ingénieur

Dans leur manière de marquer la comparaison et de générer des lectures scalaires, équatifs et similatifs fonctionnent donc clairement de façon différente.

\subsection{Scalarité et exclamation}

Une différence de fonctionnement apparaît également entre les différents types de constructions exclamatives :

\subsubsection{Exclamatives en si}

(1a) Robert est aussi grand que Maria.

(1b) Il est si grand !

Dans le cas de l'énoncé (1a), si oriente la propriété invoquée vers le haut degré mais l'orientation est bornée par les éléments au(tre) ... que, qui la positionnent sur l'échelle à un même degré que 'l'autre' (cf. $\S 3.2 .1$.). Lorsque l'étalon disparaît et avec lui les éléments de bornage dans l'opérateur (disparition du MEtal que et réduction du MRep aussi), plus rien ne limite le haut degré $(1 \mathrm{~b}:=$ «il est fort à un degré non borné »)

Le focus communicatif des comparatives en $s i+$ était centré sur la gradation du repère. Dès lors, les tours exclamatifs en si feront porter le focus communicatif exclusivement sur l'intensité de l'adjectif / de l'adverbe. La modalité intensive attribuée se cantonnant par conséquent à un niveau intra-propositionnel, cette construction se combine parfaitement avec des énoncés ayant des modes d'énonciation de type divers :

(21a) Est-ce qu'il est vraiment si fort?

(21b) Il m'a dit qu'il se sentait vraiment si fort.

(21c) Alors qu'il était si fort, une bête chute a hypothéqué ses chances de victoire.

\subsubsection{Exclamatives en comme}

(2a) Paul chante comme un rossignol.

(2a') Il chante comme ma soeur.

(2b) Comme il chante bien !

L'énoncé (2a') signifie que «Paul chante d'une manière comparable à ce que ma soeur chante ». Comme y marque la similarité entre comparé et étalon en les comparant du point de vue de la qualité évoquée et n'indique en principe pas d'orientation ou de degré sur une échelle. Toutefois, dans certains types de constructions (2a), l'exploitation de la valeur - stéréotypique ou contextuelle - de l'étalon permettait de 
produire des interprétations scalaires non inhérentes, donc discursives. A fortiori, la disparition pure et simple de l'étalon dans les constructions exclamatives ouvre la portée du marqueur qui orientait le comparé vers l'étalon et attribue dès lors la qualité en question au comparé à un niveau, au propre comme au figuré, 'incomparable'. Ceci permet d'inférer une interprétation de haut degré d'attribution de la qualité impliquée au comparé :

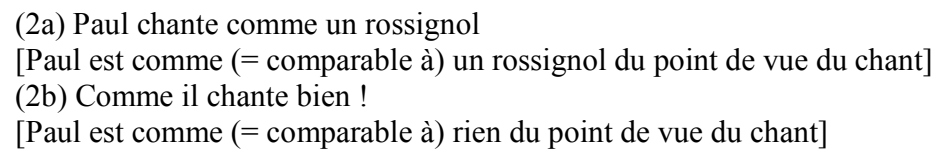

Du point de vue communicatif, rappelons que ce n'était pas la gradation de la propriété, mais bien la similarité des deux comparandes dans leur rapport à la propriété invoquée, qui est rendue saillante dans les comparatives en comme. Etant donné la disparition de tout étalon dans la construction exclamative, c'est avec une intensité sans borne que le thème (comparé sans étalon) se voit attribuer la propriété en question. Que c'est bien de l'intensité du prédicat rapporté au thème qu'il s'agit ici, sera démontré par le type de réaction qu'infèrent ces énoncés en comme :

(22a) Comme il boit, Jean !/ Oui, c'est un vrai alcoolique.

(22b) Comme vous avez de grands yeux, mère grand !/ C'est pour mieux te voir, ma petite fille.

(22c) Comme il est fort, Jean !/ Oui, c'est un véritable athlète.

Le fait que comme infère une intensité appliquée à la proposition entière explique aussi la place de comme avant le sujet (contrairement à si). Par ailleurs, il découle de ce fonctionnement que, contrairement à $s i$ une fois de plus, la construction ne se combine pas avec des énoncés de modalités différentes :

(23a) *Est-ce que vraiment comme il est fort ? / *Comme il n'est pas fort !

(23b) *Il m'a dit que comme il se sentait fort/?Il m'a dit comme il se sentait fort

(23c) *Alors que comme il était fort, une bête chute a hypothéqué ses chances de victoire.

Les seules possibilités de variation propositionnelle semblent être des tours 'exclamatifs indirects', fonctionnant sur le modèle des 'interrogatives indirectes'.

(23d) C'est fou comme il ment !

\section{Fonctionnement des marqueurs équatifs et similatifs}

\subsection{Les MRep si et si+}

$S i$ est un opérateur de scalarité (scalB) portant sur un adjectif ou un adverbe. Quel est alors la fonction des éléments complémentaires qui lui permettent d'exprimer une valeur scalA équative ?

La valeur scalA est le produit de deux éléments complémentaires, également indispensables : le préfixe aus- et le MEtal que.

\subsubsection{La complémentarité du préfixe et du MEtal.}

Si marque une orientation vers le haut degré et aus- (autre) annonce un degré égal chez un autre comparande. Toutefois, cela ne suffit pas pour fonctionner comme opérateur équatif.

En l'absence d'étalon exprimé (et du MEtal que), aussi n'exprime plus l'équativité par manque de bornage à travers un étalon mais oriente pragmatiquement à nouveau vers le haut degré (24a, reprenant $7 \mathrm{a})$ :

(24a) Elle est ressortie, toujours aussi pâle, bien que sa joue droite porte la marque rouge de cinq doigts. 
D'autre part, le préfixe semble tout aussi indispensable car l'absence du préfixe aus- rend une interprétation équative impossible (24b) et tend à orienter vers l'interprétation consécutive (24c).

Pour le fonctionnement de l'opérateur équatif, les deux composantes sont donc complémentaires : le MRep renforcé assure le positionnement sur l'échelle et le MEtal que introduit l'étalon bornant la comparaison.

\subsubsection{L'absence du préfixe aus- et l'égalité non assertée.}

Il y a un cas toutefois où la complémentarité des deux éléments assurant la valeur équative ne s'impose pas : c'est le cas des énoncés équatifs négatifs ou interrogatifs ${ }^{9}$ :

(25a) Cet exercice n'est pas si difficile qu'on le croit.

(25b) On ne l'achète pas si facilement.

(25c) Est-il vraiment si mauvais que ça ?

Comment l'expliquer? Un premier constat: le phénomène ne se limite pas au français, mais se retrouve dans de nombreuses langues : "Interestingly the older nonemphatic parameter marker often survives in negative equative constructions" (Haspelmath \& Buchholz 1998: 302). En outre, ce type d'impact semble bien dépasser les limites de la comparaison. Examinons les trois énoncés suivants ;

(26a) Paul est aussi intelligent que son frère.

(26b) Paul n'est pas si intelligent que son frère.

(26c) Paul n'est pas aussi intelligent que son frère.

(26a) pourrait être paraphrasé par /Paul est intelligent à un degré égal à son frère/. Il y a assertion d'un degré égal d'intelligence chez Paul et son frère ; l'équativité est au centre de l'énoncé et celui-ci peut être contesté dans les deux sens de l'échelle (26a': mais non, il est nettement plus intelligent que son frèrel $26 \mathrm{a} "$ : mais enfin, il est nettement moins intelligent que son frère). (26b) sera paraphrasé par/Paul n'a pas le degré d'intelligence de son frère/ dans la mesure où l'énoncé exprime de manière non ambiguë que Paul n'atteint pas le degré d'intelligence que son frère a (cf. implication = il est moins intelligent/ *il est plus intelligent). Si nous n'avons pas le marqueur $s i+$ ici, c'est tout simplement parce que ce n'est pas l'équativité qui est au centre de l'énonciation mais plutôt la non-atteinte du niveau de l'étalon. Bref il n'est plus question dans cet énoncé d'égalité et tout logiquement, l'élément soulignant l'identité avec l'autre ne s'impose plus.

Dans ce contexte, c'est (26c) qui apparaît effectivement comme moins pertinent dans la majorité des contextes dans la mesure où il centre l'énonciation sur la négation de l'équativité : /Paul est intelligent à un degré non égal à son frère/. Pragmatiquement donc, cet énoncé ne pose que la non-équativité (ou nonégalité de degré), et permet au fond des implications dans des sens divers (Paul n'est pas aussi intelligent ${ }^{10}$ que son frère, il est plus intelligent que lui).

\subsubsection{Equatives génériques et marque d'égalité.}

«Generic equatives are often formally different from specific equatives in European languages. The most typical situation in SAE languages is that the demonstrative-based parameter marker of specific equatives is lacking or is optional in generic equatives » (Haspelmath \& Buchholz $1998: 309$ ). Ce cas est illustré par les exemples du catalan (27a-b) et du néerlandais (27c-d), où nous constatons effectivement que dans les équatives générique (27b-d), le MRep est absent :

(27a) Ma sòrre es tan polida coma tu.

(27b) Es paure coma un rat de glèisa.

(27c) Paul is zo groot als zijn zuster.

(27d) Hij is sterk als een beer. 
Ce cas particulier illustre bien le jeu en français des deux marqueurs MRep et MEtal. Si nous reprenons en traduction les deux exemples catalans :

(27e) Ma sœur est aussi jolie que toi.

(27f) Il est pauvre comme un rat d'église.

nous constatons que, dans $27 \mathrm{~b} / 27 \mathrm{f}$, l'énoncé n'exprime pas un positionnement sur l'échelle (/égalité sur l'échelle de la pauvreté/) mais pose une orientation vers le haut degré, liée au caractère de parangon de l'étalon (/il ressemble à un rat d'église, question pauvreté/). En conséquence, en français, l'élément marquant l'égalité ne passe pas vers le repère, mais reste lié à MEtal, comme dans les similatives.

\subsection{Le MEtal comme}

Dans la plupart des langues d'Europe, le marqueur des similatives « is based originally on a manner relative pronoun » (Haspelmath \& Buchholz $1998: 315$ ). Cette valeur originelle continue à marquer le fonctionnement du marqueur : de proforme de manière déterminera le fonctionnement du marqueur :

- comme occupe souvent une place argumentale (de manière/ quantité) vide auprès des deux prédicats ( $\mathrm{V}$ ou Copule+Adj) dans les similatives (cf. 10a-b). Dans d'autres emplois, c'est une proforme co-saturante (2a : 'de manière similaire') ;

- même en exclamative, comme a maintenu quelques emplois révélant ses origines d'adverbe de manière (exemples de Bacha 2000) :

(28a) Comme vous y allez !

(28b) Comme vous le traitez !

Son comportement dans les exclamatives indirectes (23d) se rapproche également de celui des proformes en emploi interrogatif direct et indirect (23d') :

(23d) C'est fou comme il ment ! (Comme il ment !)

(23d') Je me demande qui ment (Qui ment?)

(23d') *C'est fou il fait si chaud (Il fait si chaud !)

- Le fonctionnement de comme avec la négation est également remarquable dans la mesure où il concentre en un seul fonctionnement syntaxo-sémantique ce que l'équatif répartit sur l'alternance aussi/ si :

(29) Paul n'est pas intelligent comme son frère.

(29a) Paul et son frère ne sont pas intelligents.

[A - intelligence et B - intelligence]

(29b) Paul n'a pas la même forme d'intelligence que son frère.

[A + intelligence et B + intelligence mais - comme]

(29c) Paul n'est pas intelligent et son frère l'est.

[A - intelligence et B + intelligence]

Notons que les interprétations (29b-c) rejoignent les effets de sens obtenus dans (26b-c) avec si ou aussi.

\section{Conclusions}

L'étude du rapport entre les constructions équatives et similatives, étendue aux constructions exclamatives voisines, montre qu'en français moderne, la propriété scalaire, qui ne doit pas être confondue avec la quantification, joue un rôle bien plus complexe qu'initialement attendu dans le départ entre les diverses constructions.

La description des différentes interprétations a mis en évidence qu'il est indispensable de distinguer non seulement diverses valeurs scalaires (scalA et scalB) mais aussi divers effets scalaires, qu'il est possible d'engendrer dans les constructions au moyen de contextes syntaxo-discursifs adéquats. En conséquence, les lectures scalaires dans les constructions examinées seront le produit de divers types de scalarité : 
Types de constructions :

\begin{tabular}{lll} 
Si+ & $\begin{array}{c}+ \text { scalA } \\
+ \text { scalB }\end{array}$ & $\begin{array}{c}+ \text { inhérent } \\
\text {-inhérent }\end{array}$ \\
\hline Si & + scalB & + inhérent \\
\hline Comme1 & -scal & + inhérent \\
& + scalA/B & -inhérent \\
\hline Comme2 & + scalB & -inhérent
\end{tabular}

En fin de compte, la scalarité ne séparerait-elle pas aussi (ou si) radicalement qu'on le prétend les constructions équatives et similatives ? Notre étude tend à montrer en effet que la propriété ne sépare pas en soi les deux constructions. Celles-ci se distinguent néanmoins par la manière dont elles instrumentalisent la scalarité :

Dans le cas des équatives, l'importance de leur lien inhérent avec la scalarité à travers leurs marqueurs se combine avec un impact syntaxo-discursif, qui permet de moduler l'expression des différentes lectures scalaires.

Pour ce qui concerne les similatives, les lectures scalaires sont essentiellement obtenus par des effets discursifs. Ceux-ci ne sont nullement inhérents aux composantes de la construction, et en particulier au marqueur comme : la similarité entre prédicats quantifiants (Il bois comme Paul), entre le comparé et un étalon générique (Il chante comme un rossignol), l'emploi d'un parangon (Il est fort comme un turc). Parfois aussi, c'est simplement l'interprétation contextuelle de l'énoncé (elle est belle comme la sœur de Jean) qui permet une interprétation scalaire. Dans le prolongement de ce fonctionnement, l'absence de tout étalon ouvre la portée du marqueur, qui orientait le comparé vers l'étalon. L'ouverture de la portée du marqueur permet d'intensifier le comparé par rapport à la qualité impliquée (comme il est fort), inférant de cette manière une lecture exclamative.

\section{Références bibliographiques}

Bacha, J. (2000). L'exclamation.Approche syntaxique et sémantique d'une modalité énonciative. L'Harmattan : Paris. Bat Zeev Shyldkrot, H. (2007). Sémantique de vrai : de l'identité à l'intensité. Travaux de linguistique, 54, 43-56.

Bolinger, D. (1972). Degree Words. Mouton: The Hague.

Buzarovska, E. (2005). Equality versus Similarity Constructions in English. Journal of Language and Linguistics, 4, $1,74-99$.

Hadermann P., Pierrard M. \& Van Raemdonck D. (2007). La scalarité, autant de moyens d'expression, autant d'effets de sens. Travaux de linguistique, 54, 7-15.

Haspelmath, M. \& Buchholz, O. (1998). Equative and similative constructions in the languages of Europe. In Van der Auwera, J. (éd.), Adverbial constructions in the languages of Europe, Mouton de Gruyter : Berlin, New York, 277-334.

Jonas, P. (1971). Les systèmes comparatifs à deux termes en ancien français. Editions de l'Université de Bruxelles : Bruxelles. 
Lehmann, C. (1989). Towards a typology of clause linkage. In Haiman, J. \& Thompson, S.A. (éds.), Clause combining in grammar and discourse, John Benjamins : Amsterdam-New York, 181-225.

Milner, J.-C. (1973). Arguments linguistiques. Mame : Paris.

Milner, J.-C. (1978). De la syntaxe à l'interprétation : quantités, insultes, exclamations. Le Seuil : Paris.

Muller, C. (1996). La subordination en français. Le schème corrélatif. Armand Colin : Paris.

Pierrard M. \& Léard J.-M. (2004). Comme : comparaison et haut degré. In M. Noailly, F. lefeuvre (éds.), Intensité, comparaison, degré, 269-286 (Travaux linguistiques du Cerlico 17).

Pierrard M. (2002). Grammaticalisation et restructuration fonctionnelle : comme et la subordination. In D. Lagorgette, P. Larrivée (éds.), Représentations du sens linguistique, LINCOM Europa: München, 293-308 (LINCOM Studies in Theoretical Linguistics 22).

Pierrard M. (1998). Comme relatif à antécédent en ancien français : grammaticalisation de la proforme indéfinie. Travaux de linguistique, 36, 127-146.

Rivara, R. (1990). Le système de la comparaison. Sur la construction du sens dans les langues naturelles. Minuit: Paris.

Rys, K. (2006). L'exclamation : assertion non stabilisée ? Le cas des exclamatives à mot qu-. Revue Romane, 41-2, 216-238.

Tovena, L. (2007). Comment devenir scalaire. Travaux de linguistique, 54, 109-119.

Zanuttini, R. \& Portner, P. (2003). Exclamative Clauses : At the Syntax-Semantics Interface. Language, 79,1, 39-81.

${ }^{1}$ Les auteurs sont cités par ordre alphabétique. Cette contribution s'inscrit dans le cadre d'une recherche financée par le FWO.

${ }^{2}$ Haspelmath \& Buchholz parlent d'un véritable «European Sprachbund characterized by a substantial set of common features, which can be called 'Standard Average European' » $(1998$ : 278).

${ }^{3} «(\ldots)$ comme renvoie à une réalité gradable, et est donc de nature intrinsèquement scalaire. C'est pourquoi son emploi dans des constructions exclamatives entraîne automatiquement l'idée de degré, (...) » (Rys $2006: 223$ ).

${ }^{4}$ Le rôle de la scalarité dans les exclamatives a été largement souligné : «Exclamatives introduce a conventional scalar implicature to the effect that the proposition they denote lies at the extreme end of some contextually given scale » (Zanuttini \& Portner 2003 : 47. Cf. aussi Rys $2006: 220$ ).

${ }^{5}$ Nous distinguons dans la comparaison à la suite de Haspelmath \& Buchholz 1998: 279 le comparé, le repère (paramètre) de la comparaison, l'étalon de comparaison, le marqueur du repère et le marqueur de l'étalon.

${ }^{6}$ La quantification est généralement entendue comme l'attribution de la quantité à un phénomène mesurable ou comptable ou la déclaration de cette quantité (trois livres; beaucoup d'eau).

${ }^{7}$ La non-disjonction référentielle du comparé et de l'étalon déclenche dans d'autres constructions aussi une lecture intensive :

(a) Distraite comme elle est, elle a oublié le jour de mon anniversaire (= elle est très distraite).

${ }^{8}$ Cette « incomplétude », produite par l'absence d'étalon, serait à la base de ce que Rys $(2006: 220)$ appelle l'effet d'instabilité de l'assertion, généré par le morphème indéfini comme, et qui est propre aux exclamatives.

${ }^{9}$ La complémentarité ne s'impose pas mais est malgré tout souvent présente :

(a) D'autres supposent que le témoignage de Micheline, tel que rapporté par Arno et Serge Klarsfeld, n'est pas aussi spontané que cela. (Le Monde, 1998).

Il s'agit donc d'une zone d'instabilité variationnelle dans le système et il s'agit d'expliquer pourquoi il y a ici cette résistance au MRep $s i+$.

${ }^{10}$ Aussi sera alors souvent marqué par un accent emphatique. 